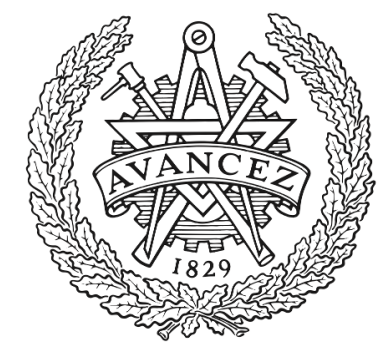

\title{
CHALMERS
}

UNIVERSITY OF TECHNOLOGY

\section{Using Two Compatible Donor Polymers Boosts the Efficiency of Ternary Organic Solar Cells to $17.7 \%$}

Downloaded from: https://research.chalmers.se, 2023-04-26 13:12 UTC

Citation for the original published paper (version of record):

Peng, W., Lin, Y., Jeong, S. et al (2021). Using Two Compatible Donor Polymers Boosts the Efficiency of Ternary Organic Solar Cells to

$17.7 \%$. Chemistry of Materials, 33(18): 7254-7262.

http://dx.doi.org/10.1021/acs.chemmater.1c01433

N.B. When citing this work, cite the original published paper. 


\title{
Using Two Compatible Donor Polymers Boosts the Efficiency of Ternary Organic Solar Cells to $17.7 \%$
}

\author{
Wenhong Peng, Yuanbao Lin,* Sang Young Jeong, Yuliar Firdaus, Zewdneh Genene, Aggelos Nikitaras, \\ Leonidas Tsetseris, Han Young Woo, Weiguo Zhu,* Thomas D. Anthopoulos, * and Ergang Wang*
}

Cite This: Chem. Mater. 2021, 33, 7254-7262

Read Online

\section{ACCESS 1}

Џ Metrics \& More

Article Recommendations

Supporting Information

ABSTRACT: The use of ternary organic semiconducting blends is recognized as an effective strategy to boost the performance of polymer solar cells (PSCs) by increasing the photocurrent while minimizing voltage losses. Yet, the scarcity of suitable donors with a deep highest occupied molecular orbital (HOMO) level poses a challenge in extending this strategy to ternary systems based on two polymers. Here, we address this challenge by the synthesis of a new donor polymer (PM7-Si), which is akin to the well-known PM6 but has a deeper HOMO level. PM7-Si is utilized as the third component to enhance the performance of the best-in-class binary system of PM6:BTP-eC9, leading to simultaneous improvements in the efficiency $(17.7 \%)$, open-circuit voltage $(0.864 \mathrm{~V})$, and fill

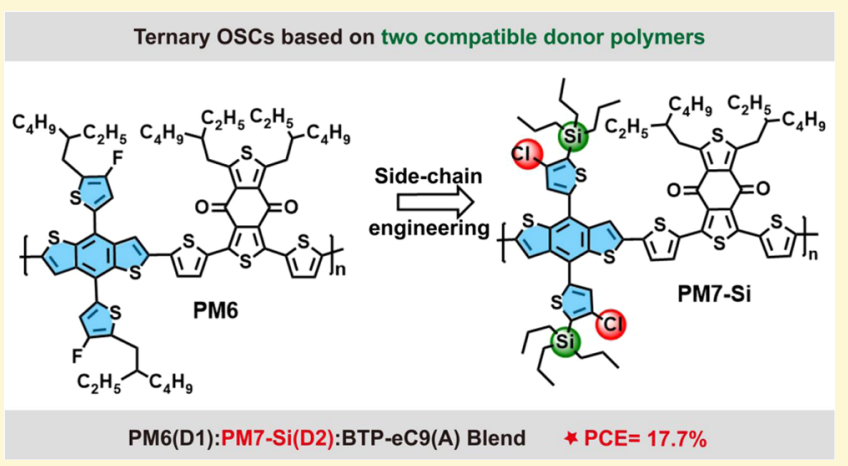
factor $(77.6 \%)$. These decisively enhanced features are attributed to the efficient carrier transport, improved stacking order, and morphology. Our results highlight the use of two polymer donors as a promising strategy toward high-performance ternary PSCs.

\section{INTRODUCTION}

Polymer solar cells (PSCs) represent an attractive technology due to their advantageous mechanical properties, tunable optical properties, controllable morphology, and suitability for the fabrication of large-area devices via upscalable printing techniques. $^{1-7}$ The very recent development of the nonfullerene acceptor $\mathrm{Y}^{\prime} \mathrm{s}^{8,9}$ derivative, namely, BTP-eC9 (abbreviated as C9 in this work), has boosted the power conversion efficiency (PCE) of PSCs to over 18\%, thanks to its suitable energetics and enhanced electron transport properties than Y6. ${ }^{10-14}$ However, the low-lying lowest unoccupied molecular orbital (LUMO) of Y6 and its derivatives results in PSCs with a relatively low open-circuit voltage $\left(V_{o c}\right)$ even when they are blended with polymer donors with deep highest occupied molecular orbital (HOMO) levels. ${ }^{15-22}$ Among the latter, PM6 is currently one of the most widely used polymer donor to pair with Y6 derivatives. Its outstanding absorption spectra, high carrier mobility, and the deep HOMO level ${ }^{1}$ have resulted in cells with PCEs of over $17 \%,{ }^{23,24}$ even though the achieved $V_{\text {oc }}(\sim 0.84 \mathrm{eV})$ remains suboptimal. ${ }^{10-12,15}$

To date, the ternary blend strategy represents the most promising approach for increasing the $V_{\mathrm{oc}}$ of PSCs, ${ }^{25,26}$ and significant effort has been dedicated to understanding the key parameters that determine the key operating parameters. ${ }^{27-31}$ Studies have shown that selecting an appropriate third component (guest donor D or acceptor A) can increase the $V_{\text {oc }}$ by optimizing the alignment of energy levels. Typical active-layer structures of ternary cells are divided into "D:A1:A2" (1D/2A) and "D1:D2:A" (2D/1A) systems. Narrow band-gap nonfullerene acceptors have been widely used as guest acceptors in a D:A1:A2 system primarily to improve the collection of light in the active layer by extending its absorption spectra. Although very simple, this approach has helped to boost the PCE but with only a marginal increase of the cell's $V_{\text {oc }}{ }^{25,26}$ For example, several ternary PSCs with a $\mathrm{D}: \mathrm{A} 1: \mathrm{A} 2$ structure have recently reached a PCE value of over $18 \% .{ }^{32,33}$ On the other hand, only a few highly efficient (PCE $>16 \%)$ ternary PSCs based on a D1:D2:A blend have been reported thus far, mainly because of the lack of suitable polymer donors (Table S1, Supporting Information). ${ }^{34-36}$ In particular, ternary systems with two compatible polymer donors have been rarely studied using the best-in-class PM6:C9 system. The similarity of the PM6 and C9 HOMO levels $\left(-5.50^{37}\right.$ and $-5.64 \mathrm{eV},{ }^{10}$ respectively) makes it difficult to design a third component with an energy level somewhere between these two. Apart from the requirement of suitable energy levels, structural similarity with the host donor is also

Received: April 25, 2021

Published: September 8, 2021 
(a)

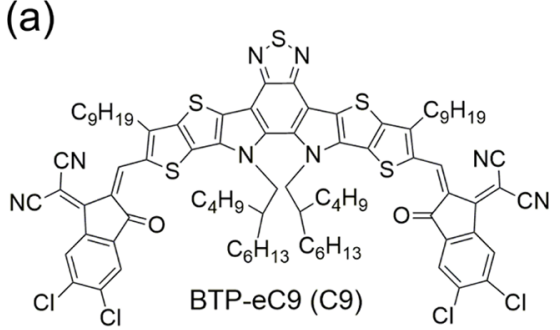

(b)

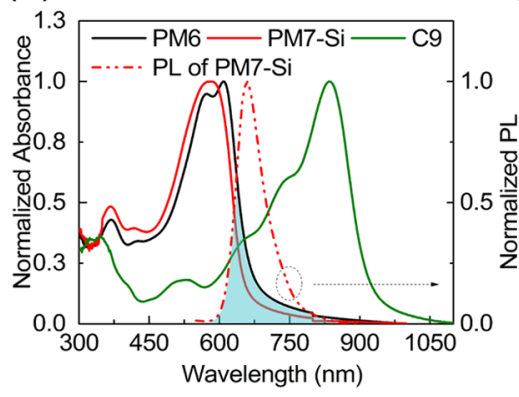

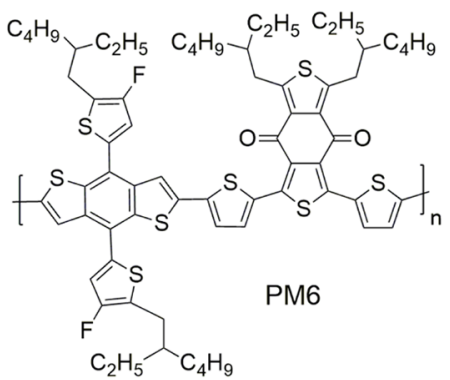

(c)

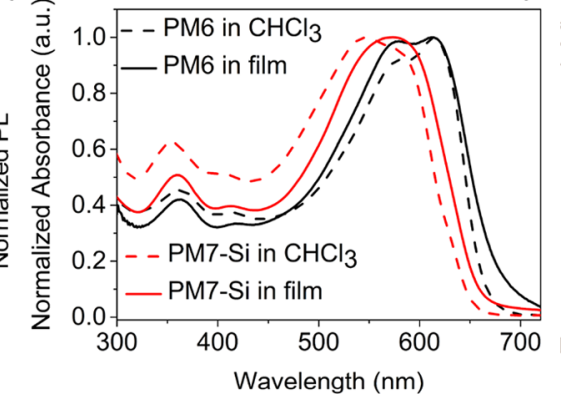

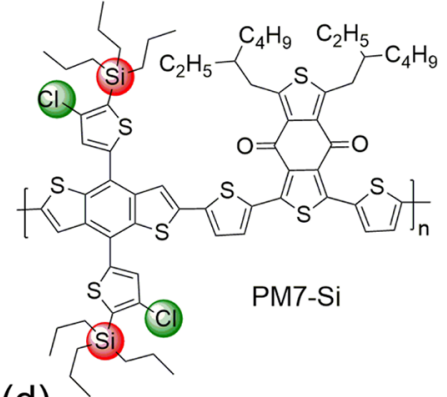

(d)

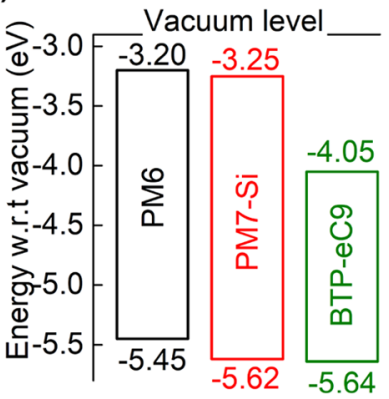

Figure 1. (a) Chemical structures of BTP-eC9 (C9), PM6, and PM7-Si. (b) Optical absorption spectra of PM6, PM7-Si, and C9 neat films, as well as the normalized photoluminescence (PL) emission spectrum of the neat PM7-Si film. (c) Normalized UV-vis absorbance spectra of PM6 and PM7-Si in $\mathrm{CHCl}_{3}$ and the film. (d) Energy levels of PM6, PM7-Si, and C9.

required for the sake of better miscibility and optimized morphology in the bulk heterojunction (BHJ) so as to augment $V_{\text {oc }}$ without sacrificing the short-circuit current density $\left(J_{\text {sc }}\right)$, the fill factor $(\mathrm{FF})$, and the PCE. ${ }^{38-41}$

Previous studies have identified the promising role of chlorine $(\mathrm{Cl})$ atoms and alkylsilyl side chains in enhancing the voltage and photovoltaic properties of two-dimensional (2D)conjugated polymers. ${ }^{4-44}$ Based on the well-known polymer PM6 and side-chain engineering, herein, we designed and synthesized a donor-acceptor (D-A)-type polymer named PM7-Si by replacing fluorine with chlorine and the ethylhexyl groups with alkylsilyl chains. The larger dipole moments of the carbon $(\mathrm{C})$-chlorine $(\mathrm{Cl})$ bonds of the thiophene conjugated side chains, as compared to carbon (C)-fluorine (F) bonds, combined with the $\sigma^{*}(\mathrm{Si})-\pi^{*}(\mathrm{C})$ bond interactions, downshift the HOMO level of PM7-Si relative to that of PM6. To test our hypothesis, we added PM7-Si into the PM6:C9 blend and fabricated PSCs. Cells containing $10 \mathrm{wt} \%$ PM7-Si yield optimal performance with a PCE value of $17.7 \%, V_{\text {oc }}$ of 0.864 $\mathrm{V}, J_{\mathrm{sc}}$ of $26.05 \mathrm{~mA} \mathrm{~cm}{ }^{-2}$, and an FF of $77.6 \%$. The PCE of control cells based on the binary PM6:C9 system remained significantly lower $(\approx 17 \%)$. To this end, we note that the achieved PCE of $17.7 \%$ is among the highest values for ternary PSCs based on the D1:D2:A system reported to date (Table S1). The improved performance was attributed to the structural affinity of the two donors, which, in turn, provides a high quality for the morphology of the ternary blend while reducing bimolecular recombination. In addition, the low-lying HOMO level of PM7-Si improves the energy level alignment, leading to a significant increase in the $V_{\text {oc }}$ without compromising the $J_{\mathrm{sc}}$.

\section{EXPERIMENTAL SECTION}

Materials. PFN-Br, PM6, and BTP-eC9 were bought from Solarmer Materials Inc. PM7-Si was designed and synthesized in this work, as shown in Scheme S1. All reactions for the preparation of PM7-Si were carried out in oven-dried glassware under a nitrogen atmosphere, and all solvents were used as commercially supplied unless otherwise stated.

General Characterization. ${ }^{1} \mathrm{H}$ and ${ }^{13} \mathrm{C}$ nuclear magnetic resonance (NMR) spectra of all compounds were recorded on a Bruker AV $400 \mathrm{MHz}$ Fourier transform (FT)-NMR spectrometer using $\mathrm{CDCl}_{3}$ as a solvent. Molecular weights of polymer PM7-Si were measured by high-temperature gel permeation chromatography (GPC) using monodispersed polystyrene as a standard and 1,2,4trichlorobenzene as an eluent at $150{ }^{\circ} \mathrm{C}$. Ultraviolet-visible (UV-vis) spectra were recorded on a UV-vis-near-infrared (NIR) spectrophotometer of Agilent Technologies Cary Series. Electrochemical cyclic voltammetry (CV) was taken on a $\mathrm{CH}$-Instruments 650A Electrochemical Workstation in three electrodes using a glassy carbon as a working electrode, a $\mathrm{Pt}$ wire as a counter electrode, and a $\mathrm{Ag} / \mathrm{Ag}^{+}$ reference electrode calibrated using a ferrocene/ferrocenium $(\mathrm{Fc} /$ $\mathrm{Fc}^{+}$) redox couple. Tetrabutylammonium hexafluorophosphate $\left(\mathrm{Bu}_{4} \mathrm{NPF}_{6}\right)(0.1 \mathrm{M})$ in an anhydrous acetonitrile solution was used as an electrolyte, which was bubbled with nitrogen before each measurement. The HOMO and LUMO energies and plots were obtained with density functional theory (DFT) calculations using the NWChem code, ${ }^{45}$ the hybrid B3LYP ${ }^{46,47}$ exchange-correlation (xc) functional, and the DZVP orbital basis. ${ }^{48}$ Structures and HOMO/ LUMO plots were rendered with VESTA. ${ }^{49}$ The reported HOMO energies are the results of the DFT calculations. LUMO energies, on the other hand, were obtained by adding to the HOMO energies the energy difference between the ground state and the first excited singlet state. We have calculated this energy difference using the Tamm-Danncoff (TDA) version of time-dependent DFT. ${ }^{50}$

\section{RESULTS AND DISCUSSION}

The molecular structures of BTP-eC9 (C9), PM6, and PM7-Si are shown in Figure 1a. PM7-Si was synthesized by the Stille coupling polymerization between ((2,6-bis(trimethylstannyl)benzo[ $\left.1,2-b: 4,5-b^{\prime}\right]$ dithiophene-4,8-diyl)bis(3-chlorothiophene-5,2-diyl))bis(tripropylsilane) (M1) and 1,3-bis(5-bromothiophen-2-yl)-5,7-bis(2-ethylhexyl)-4H,8H-benzo[1,2$\left.c: 4,5-c^{\prime}\right]$ dithiophene-4,8-dione (M2), as shown in Scheme S1 and Figures S1-S5. High-temperature gel permeation chromatography (GPC), as shown in Figure S6, was employed 
(a)
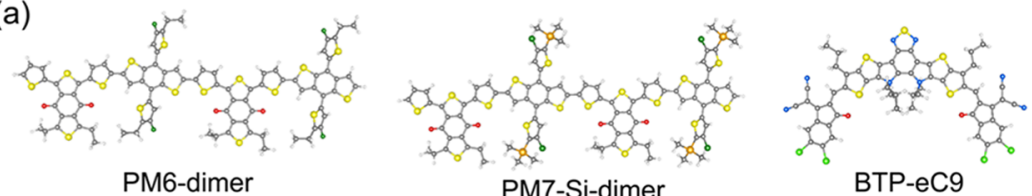

(b)
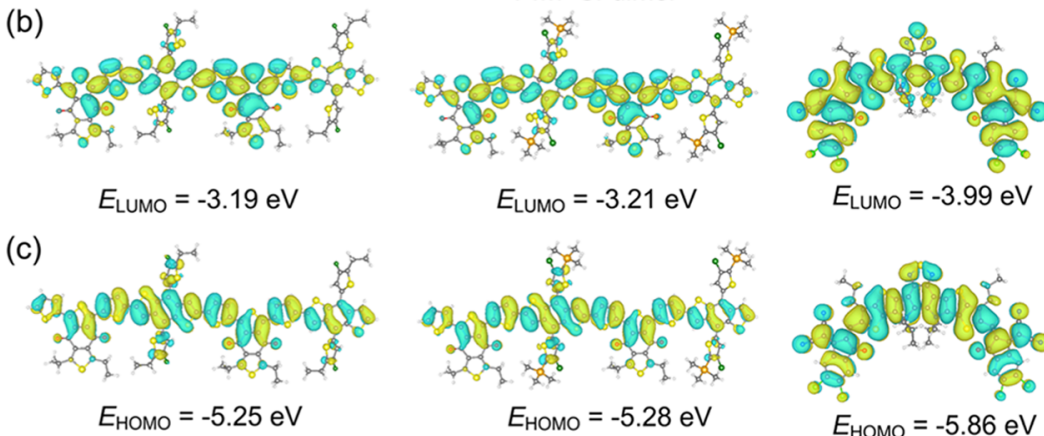

$E_{\mathrm{HOMO}}=-5.25 \mathrm{eV}$

$E_{\text {HOMO }}=-5.28 \mathrm{eV}$

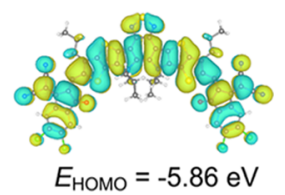

Figure 2. (a) Minimum energy backbone conformations, (b) LUMO and (c) HOMO energy levels of PM6, PM7-Si dimers, and acceptor C9 (C: gray, Si: orange, S: yellow, O: red, F and $\mathrm{Cl}$ : green, $\mathrm{H}$ : white spheres) calculated by the DFT method.

using 1,2,4-trichlorobenzene as an eluent to estimate the number average molecular weight $\left(M_{\mathrm{n}}\right) /$ polydispersity index (PDI), which turns out to be $16.3 \mathrm{kDa} / 3.20$ (Table S2). The polymer presents good solubility in organic solvents such as chloroform (CF), chlorobenzene, and $o$-dichlorobenzene.

To assess the optical response of the systems of interest, we measured their ultraviolet-visible (UV-vis) absorption spectra. As can be seen, the UV-vis spectra of PM6 and PM7-Si films are complementary to that of C9 (Figure 1b), which is beneficial with regard to light-harvesting. In addition, the normalized absorption spectra of PM6 and PM7-Si in a CF solution and thin films are shown in Figure 1c, and the detailed photophysical properties are given in Table S3. PM7-Si exhibits an obviously blue-shifted absorption band compared to that of PM6 due to the introduction of the weak electronwithdrawing chlorine $(\mathrm{Cl})$ atoms and alkylsilyl as side chains on the benzodithiophene (BDT) unit in PM7-Si, which is valuable for further improving the light collection of the ternary blend by altering the content of PM7-Si. Thereby, the optical band gap of PM7-Si $(1.88 \mathrm{eV})$ is slightly wider than that of PM6 $(1.82 \mathrm{eV})$. It is worth noting that the photoluminescence (PL) spectrum of PM7-Si is slightly overlapped with the absorption spectrum of PM6 (see Figure $1 \mathrm{~b})$, indicating that there is possible energy transfer between PM6 and PM7-Si. To verify this, the PL spectrum of neat PM6, PM7-Si, and their blend films were investigated (Figure S7). The neat PM7-Si film shows an intense PL emission at $660 \mathrm{~nm}$, while the neat PM6 film exhibits a relatively weak emission intensity at $680 \mathrm{~nm}$. After blending PM6 with a small amount of PM7-Si, we can see that the characteristic PL peak of PM7-Si completely disappeared with an obviously enhanced emission of PM6, indicating the energy transfer from PM7-Si to PM6. ${ }^{51}$

The HOMOs/LUMOs of the two polymers were determined by cyclic voltammetry $(\mathrm{CV})$ measurements in thin films, as shown in Figure S8 and Table S3. As depicted in the energy diagram of Figure 1d, the HOMO and LUMO energy levels of PM7-Si lie between those of PM6 and C9, a desired feature for efficient charge transfer in the ternary blend. Moreover, the low-lying HOMO of PM7-Si increases the offset between the HOMO of the donor and the LUMO of the acceptor, which is expected to result in a higher $V_{\text {oc }}$ in the ternary PSC device. $^{52-54}$ It should also be noted that the HOMO-HOMO offset between the donor and the acceptor is relatively small, but, as a recent study has shown, this offset does not have to be large for efficient PSC devices. ${ }^{55}$ The experimental HOMO/LUMO values were corroborated by density functional theory (DFT) calculations of the energies of the frontier orbitals of PM6, PM7-Si, and C9. To expedite the calculations, without sacrificing any accuracy, all long alkyl chains were replaced by methyl groups and polymers were modeled as dimers. As displayed in Figure 2 and Table S3, the calculated HOMO/LUMO energy levels of PM6, PM7-Si, and C9, are, respectively, $-5.25 /-3.19,-5.28 /-3.21$, and -5.86 / $-3.99 \mathrm{eV}$. Hence, both theoretical calculations and experimental data consistently support the result that the introduction of the $\mathrm{Cl}$ atom and the alkylsilyl side chain has a positive impact on slightly deepening the HOMO energy levels of polymer donors.

To investigate the compatibility of the used materials, the contact angles for deionized water $\left(\mathrm{H}_{2} \mathrm{O}\right)$ and formamide $\left(\mathrm{HCONH}_{2}\right)$ were measured on PM6, PM7-Si, and C9 neat films, as shown in Figure $\mathrm{S} 9$. The water $/ \mathrm{HCONH}_{2}$ contact angles of PM6, PM7-Si, and C9 were 100.4/82.4, 105.8/83.8, and $94.3 / 70.8^{\circ}$, respectively (Table 1 ). According to the

Table 1. Summary of Contact Angles of Water and Glycerol and Surface Tension of PM6, PM7-Si, and c9 Neat Films, and $\chi$ Values of Binary Blends

\begin{tabular}{lrcclc}
$\begin{array}{l}\text { neat } \\
\text { film }\end{array}$ & $\begin{array}{r}\theta_{\text {water }} \\
{[\mathrm{deg}]}\end{array}$ & $\begin{array}{c}\theta_{\mathrm{HCONH} 2} \\
{[\mathrm{deg}]}\end{array}$ & $\begin{array}{c}\gamma \\
{\left[\mathrm{mN} \mathrm{m}^{-1}\right]}\end{array}$ & binary blend & $\chi$ \\
PM6 & 100.4 & 82.4 & 20.3 & PM6:C9 & 0.86 \\
PM7-Si & 105.8 & 83.8 & 22.3 & PM7-Si:C9 & 0.50 \\
C9 & 94.3 & 70.8 & 29.5 & PM6:PM7-Si & 0.05 \\
\hline
\end{tabular}

contact angle data, the surface tensions $(\gamma)$ calculated using the Owens, Wendt, Rabel, and Kaelble (OWRK) equation ${ }^{56}$ are summarized in Table 1 . The close $\gamma$ values of PM6 and PM7-Si indicate their good miscibility. Furthermore, the FloryHuggins interaction parameter $(\chi)$ value of the binary blend can be obtained using the formula shown as follows: ${ }^{57}$ $\chi_{\mathrm{A}-\mathrm{B}} \propto\left(\sqrt{\gamma_{\mathrm{A}}}-\sqrt{\gamma_{\mathrm{B}}}\right)^{2}$, where $\gamma_{\mathrm{A}}$ and $\gamma_{\mathrm{B}}$ are the surface energy values of components $A$ and $B$ calculated from the 
(a)
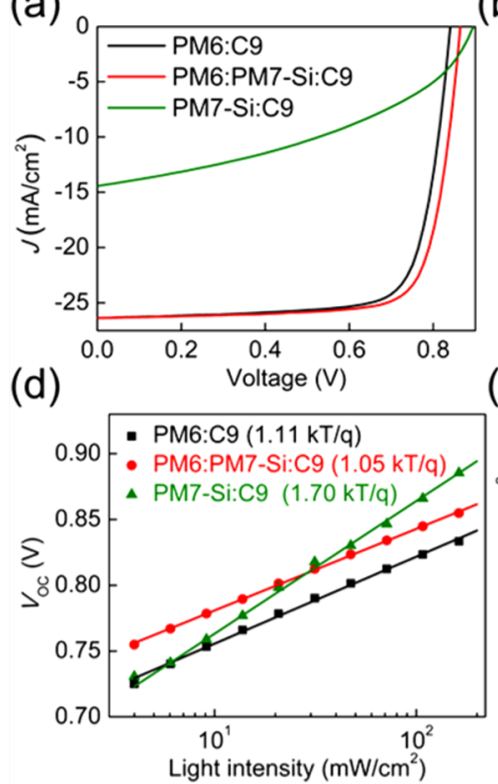

(b)

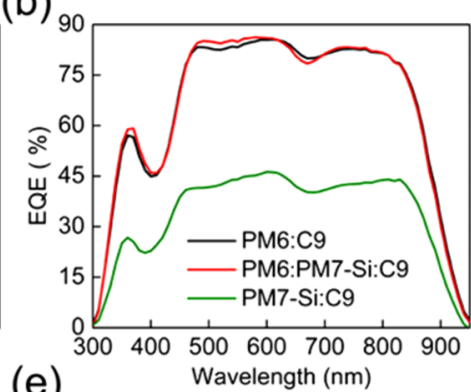

(e)

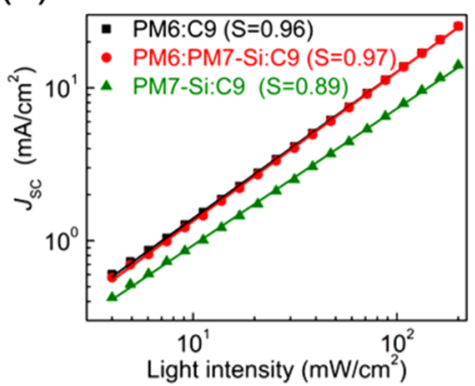

(c)

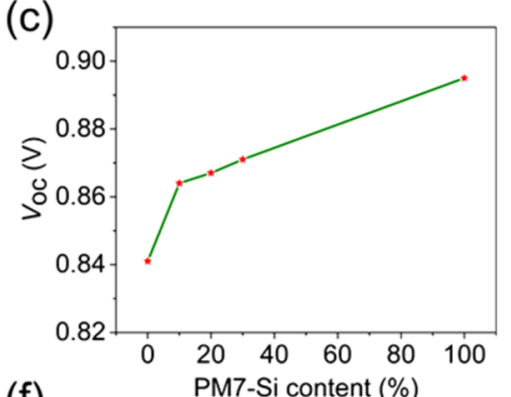

(f)

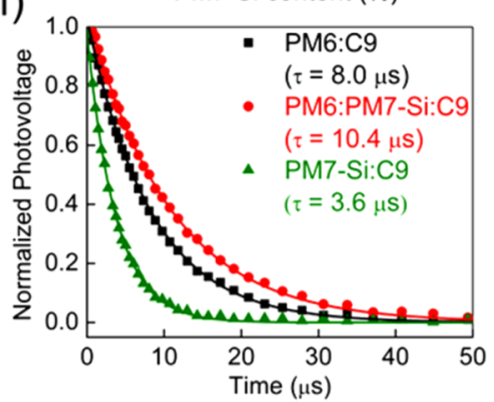

Figure 3. (a) $J-V$ and (b) external quantum efficiency (EQE) curves of optimized devices based on PM6:C9 = 1:1.2 (w/w), PM6:PM7-Si:C9 = 0.9:0.1:1.2 (w/w), and PM7-Si:C9 = 1:1.2 (w/w) BHJ films. (c) $V_{\text {oc }}$ values of PM6:PM7-Si:C9 devices containing different PM7-Si weight ratios. (d) Dependence of $V_{\mathrm{oc}}$ on the light intensity, (e) dependence of $J_{\mathrm{sc}}$ on the light intensity, and (f) transient photovoltage (TPV) spectra for the optimized binary and ternary PSC devices.

Table 2. Device Parameters of Ternary PSCs with Varying PM7-Si Contents ${ }^{a}$

$\begin{array}{cccccc}\text { PM6:PM7-Si:C9 } & V_{\text {oc }}[\mathrm{V}] & J_{\mathrm{sc}}\left[\mathrm{mA} \mathrm{cm}^{-2}\right] & \text { Calc. } \mathrm{J}_{\mathrm{sc}}\left[\mathrm{mA} \mathrm{cm}^{-2}\right] & \mathrm{FF}[\%] & \text { PCE }_{\max }\left(\mathrm{PCE}_{\text {avg }}\right)[\%] \\ 1: 0: 1.2 & 0.841 & 26.36 & 26.07 & 76.6(75.5 \pm 0.7) & 17.0(16.6 \pm 0.2) \\ 0.9: 0.1: 1.2 & 0.864 & 26.35 & 26.05 & 77.6(76.6 \pm 0.7) & 17.7(17.3 \pm 0.2) \\ 0: 1: 1.2 & 0.895 & 14.43 & 14.33 & 41.7(38.8 \pm 2.2) & 5.4(5.2 \pm 0.1)\end{array}$

${ }^{a}$ Average values obtained from 15 different cells.

contact angle measurements, respectively. The $\chi$ values for PM6:C9 and PM7-Si:C9 are 0.86. and 0.50, respectively. The higher $\chi$ value of PM6:C9 blend reflects its possibly higher domain purity, which can suppress the bimolecular recombination in blends and further promote the photons to convert into excitons more efficiently.

Binary and ternary PSCs with a device configuration of an ITO/PEDOT:PSS $(\approx 40 \mathrm{~nm}) /$ active layer $(110 \mathrm{~nm}) / \mathrm{PFN}$ $\operatorname{Br}(5 \mathrm{~nm}) / \mathrm{Ag}(100 \mathrm{~nm})$ were fabricated to evaluate the photovoltaic performance of the various blend compositions. The current density-voltage $(J-V)$ curves of the PSCs are shown in Figures 3a and S10, and the detailed photovoltaic parameters are summarized in Tables 2 and S4. The binary PM6:C9-based PSCs yield a PCE of $17.0 \%$, with a $V_{\text {oc }}$ of 0.841 $\mathrm{V}$, a $J_{\mathrm{sc}}$ of $26.36 \mathrm{~mA} \mathrm{~cm}^{-2}$, and $\mathrm{FF}$ of $76.6 \%$, in good agreement with previous reports. ${ }^{10}$ On the other hand, PSCs based on the binary PM7-Si:C9 blend exhibit enhanced $V_{\text {oc }}(0.895 \mathrm{~V})$ due to the lower HOMO of PM7-Si but with significantly reduced PCE (5.4\%), $J_{\mathrm{sc}}\left(14.43 \mathrm{~mA} \mathrm{~cm} \mathrm{~cm}^{-2}\right)$, and FF (41.7\%) values. Optimal performance for the ternary cells was obtained when $10 \%$ of PM7-Si, as the guest donor, was incorporated into PM6:C9 blends. These ternary PSCs exhibited superior performance with a clearly increased PCE (17.7\%) owing to the simultaneously increased $V_{\mathrm{oc}}(0.864 \mathrm{~V})$ and FF $(77.6 \%)$, with $J_{\mathrm{sc}}$ remaining largely unchanged $\left(26.35 \mathrm{~mA} \mathrm{~cm}{ }^{-2}\right)$ as compared to binary PM6:C9-based PSCs.

The results show that PM7-Si acts as a promising third component for the ternary PSCs based on PM6 and C9. Its positive effect is better illustrated in the increasing $V_{\text {oc }}$ with increasing PM7-Si concentration in the ternary blend (Figure $3 c$ ). It is also found that the $V_{\text {loss }}$ values of ternary organic solar cells (OSCs) were gradually reduced along with the increment of the PM7-Si content (Table S4). We utilized the time-offlight secondary-ion mass spectrometry (TOF-SIMS) to detect the vertical distribution of the composition in $\mathrm{BHJ}$, as presented in Figure S11. In a binary active layer (PM6:BTPeC9), the PM6 donor is rich near the top surface, while the BTP-eC9 acceptor is rich near the bottom region of the $\mathrm{BHJ}$ layer, which is in agreement with previous reports. ${ }^{58,59}$ Moreover, the addition of a PM7-Si guest donor plays a negligible role in the distribution of the composition in a ternary system, showing that donors are rich on the top surface and acceptors are rich on the bottom surface. Hence, a nonuniform vertical phase separation exists in both binary and ternary blends and it is not clearly related to the tendency of $V_{\text {oc }}$ variation. Clearly, these results indicate that the combination of two compatible polymer donors with a suitable acceptor system provides a viable route toward improved $V_{\text {oc }}$ and thus cell performance. ${ }^{54,60-62}$ The external quantum efficiency (EQE) spectra of the optimized PSC devices are shown in Figure 3b, whereby the PM6:C9 binary PSCs and PM6:PM7-Si:C9 ternary PSCs exhibit a similar EQE response between 300 and $930 \mathrm{~nm}$. As listed in Table 2, the integrated $J_{\text {sc }}$ based on the EQE spectrum matches well with the measured $J_{\mathrm{sc}}$ value from $J-V$ curves $( \pm 3 \%)$. In contrast, the PM7-Si:C9 device shows a relatively poor photoelectric 
(a)
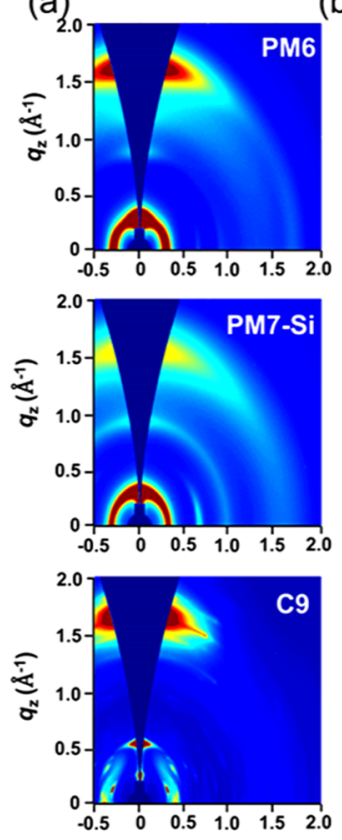

$q_{\mathrm{xy}}\left(\AA^{-1}\right)$ (b)

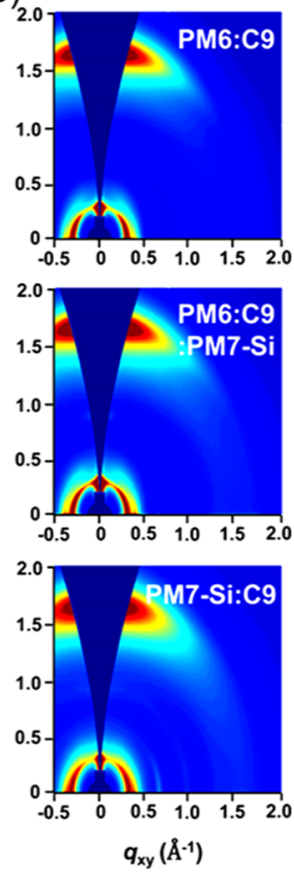

(c)

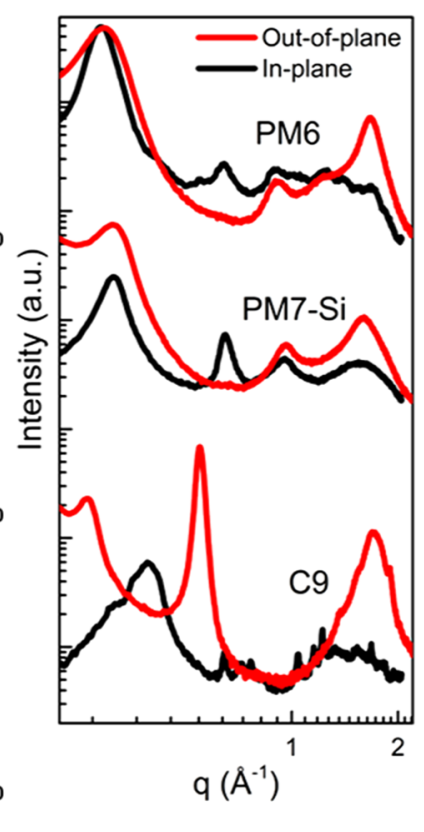

(d)

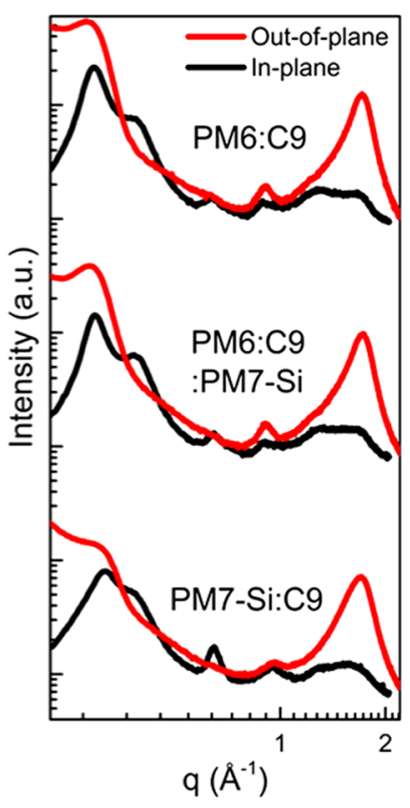

Figure 4. (a, b) 2D GIWAXS patterns and (c, d) scattering profiles for neat PM6, PM7-Si, and C9 and for PM6:C9 = 1:1.2 (w/w), PM6:PM7Si:C9 = 0.9:0.1:1.2 (w/w), and PM7-Si:C9 = 1:1.2 (w/w) BHJ films.

response with a maximum $\mathrm{EQE}$ value of $\approx 45 \%$ and a low integrated current density of $14.43 \mathrm{~mA} \mathrm{~cm}^{-2}$, which is ascribed to the inefficient charge separation in the blend.

To further understand the charge carrier recombination mechanism in these devices, we probed the dependence of $V_{\mathrm{oc}}$ and $J_{s c}$ on light intensity $\left(P_{\text {light }}\right)$. Based on the corresponding results shown in Figures 3d,e, the relationship between $J_{s c}$ and $P_{\text {light }}$ can be described by the formula $J_{\text {sc }} \propto P_{\text {light }}{ }^{\alpha}$, where the value of the exponent $\alpha$ gauges the degree of bimolecular recombination. $^{63,64}$ Generally, a value for $\alpha$ close to 1 means that the bimolecular recombination in the donor/acceptor (D/ A) interface is negligible. In the present case, we derive $\alpha$ values of 0.96 and 0.89 for the PM6:C9 and PM7-Si:C9 binary devices, respectively. The parameter $\alpha$ approaches $1(\alpha=0.97)$ upon $10 \mathrm{wt} \%$ addition of PM7-Si into the PM6:C9 blend, indicating reduced bimolecular recombination in the ternary device. Likewise, the relationship was examined between the $V_{\mathrm{oc}}$ and $P_{\text {light }}$ by fitting the equation of $V_{\mathrm{oc}} \propto n k_{\mathrm{B}} T / q \ln \left(P_{\text {light }}\right)$, where $k_{\mathrm{B}}$ is Boltzmann's constant, $T$ is the temperature (in $\mathrm{K}$ ), and $q$ is the elementary charge. ${ }^{65,66}$ The slope values were calculated to be $1.11,1.70$, and $1.05 \mathrm{kT} / \mathrm{q}$ for the PM6:C9, PM7-Si:C9, and PM6:PM7-Si:C9 devices, respectively. Clearly, monomolecular recombination appears to dominate the operation of PM7-Si:C9 cells, leading to the poor performance observed. The slope values measured for PM6:C9 and PM6:PM7-Si:C9-based devices, on the other hand, were close to $1 k_{B} T / q$, indicating minimal recombination loses. In fact, the slope of ternary PSCs decreased slightly with the addition of PM7-Si, which implies less monomolecular recombination in the ternary cell. Overall, these results suggest that both monomolecular and bimolecular recombination are effectively suppressed in the ternary devices, boosting their FF and PCE.

The recombination losses in both the ternary and binary blend PSCs were further studied using transient photovoltage (TPV) measurements (Figure 3f). The recombination time $\left(\tau_{\text {rec }}\right)$ of photocarriers in PM6:C9 and PM7-Si:C9 devices was found to be 8.0 and $3.6 \mu \mathrm{s}$, respectively. The prolonged lifetime in the device implies that the improved charge transport pathway suppresses carrier recombination effectively, and the diminished recombination is beneficial to $J_{s c}$ enhancement. ${ }^{67}$ We found that the $\tau_{\text {rec }}$ for the PM6:PM7-Si:C9 ternary device is significantly longer $(10.4 \mu \mathrm{s})$ than those in the binary blend devices, implying smaller recombination of photocarriers in agreement with the aforementioned analysis. Overall, the results on charge recombination are consistent with the improved $J_{\mathrm{sc}}$ and PCE in optimized ternary PSCs.

The hole $\left(\mu_{\mathrm{h}}\right)$ and electron $\left(\mu_{\mathrm{e}}\right)$ mobilities were also evaluated by the space-charge-limited current (SCLC) method using hole-only and electron-only device architectures composed of ITO/PEDOT:PSS/BHJ/ $\mathrm{MoO}_{3} / \mathrm{Ag}$ and ITO/ $\mathrm{ZnO} / \mathrm{BHJ} / \mathrm{PFN}-\mathrm{Br} / \mathrm{Al}$, respectively. The resulting $J-V$ curves and charge carrier mobilities of the neat and blend films are presented in Figure S12 and Tables S5, S6. The neat PM6 film shows $\mu_{\mathrm{h}}$ of $1.7 \times 10^{-4} \mathrm{~cm}^{2} \mathrm{~V}^{-1} \mathrm{~s}^{-1}$, while the neat C9 film exhibits $\mu_{\mathrm{e}}$ of $2.9 \times 10^{-4} \mathrm{~cm}^{2} \mathrm{~V}^{-1} \mathrm{~s}^{-1}$, which are in agreement with results reported previously. ${ }^{1,41}$ The hole mobility of the PM7-Si film $\left(2.8 \times 10^{-5} \mathrm{~cm}^{2} \mathrm{~V}^{-1} \mathrm{~s}^{-1}\right)$ is lower than that of PM6 because of its relatively lower crystallinity, as confirmed by the grazing-incidence wide-angle X-ray scattering (GIWAXS) measurements that will be discussed next. For the optimal binary and ternary blends, the $\mu_{\mathrm{e}}$ values are of the same order of magnitude $\left((1.7-2.8) \times 10^{-4} \mathrm{~cm}^{2} \mathrm{~V}^{-1} \mathrm{~s}^{-1}\right)$, but the $\mu_{\mathrm{h}}$ value of the binary PM7-Si:C9 blend differs significantly from the others mainly due to the low hole mobility of the neat PM7-Si film. The addition of 10\% PM7-Si into the PM6:C9 blend leads to a more balanced and enhanced charge transport, which most likely underpins the improved FF measured for optimized ternary PSCs. These improvements most likely relate to an improved active-layer morphology induced by PM7-Si and will be discussed next. 


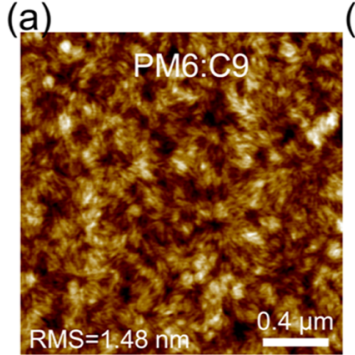

(d)

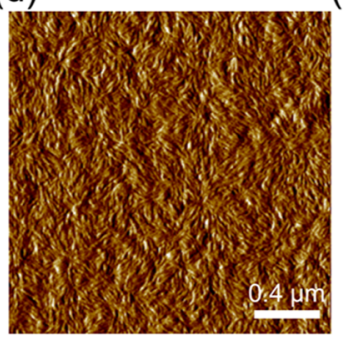

(b)

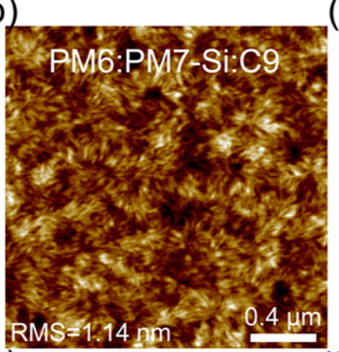

(e)

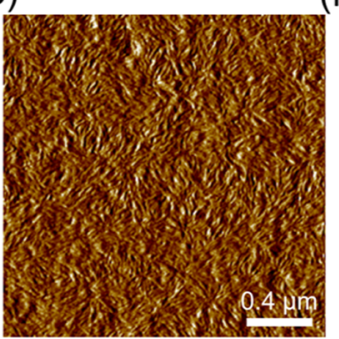

(c)

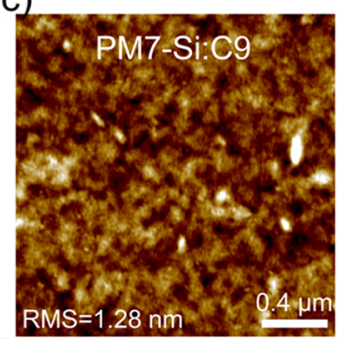

(f)

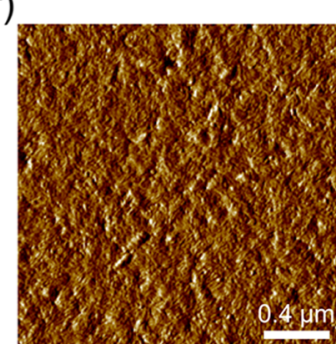

Figure 5. $(\mathrm{a}-\mathrm{c})$ Tapping mode AFM topography and $(\mathrm{d}-\mathrm{f})$ phase images of PM6:C9 = 1:1.2 (w/w) BHJ films, PM6:PM7-Si:C9 = 0.9:0.1:1.2 (w/ w) BHJ films, and PM7-Si:C9 = 1:1.2 (w/w) BHJ films. The size of the AFM image is $2 \mu \mathrm{m} \times 2 \mu \mathrm{m}$.

The molecular packing and crystallinity in both pure and blend films were investigated by grazing-incidence wide-angle X-ray scattering (GIWAXS) measurements. Figure 4 displays the two-dimensional (2D) GIWAXS patterns and scattering profiles of pure and blend films. The corresponding parameters are summarized in Tables S7 and S8. The PM6 film exhibits a face-on dominant orientation with a strong $\pi-\pi$ stacking (010) peak at $q_{z}=1.67 \AA^{-1}(d$-spacing of $3.76 \AA)$ along the out-ofplane (OOP) direction. Different from PM6, PM7-Si shows a much weaker (010) scattering at $1.60 \AA^{-1}$ ( $d$-spacing of 3.93 $\AA)$ in the OOP direction, indicating a reduced degree of crystallinity. This result indicates that the side-chain modification significantly alters the packing characteristics of the polymer, which can also support the observed low hole mobility from the PM7-Si film. On the other hand, the C9 film demonstrates the preferred face-on orientation showing more prominent $(010)$ diffraction peaks at $q \approx 1.70 \AA^{-1}$ ( $d$-spacing of $3.70 \AA$ ) in the OOP direction. Moreover, the morphology of the PM6:PM7-Si:C9 blend film is not disrupted by the incorporation of $10 \% \mathrm{PM} 7-\mathrm{Si}$ as compared to PM6:C9, indicating compatibility of the two donor polymers. The good compatibility of the two donor polymers is also supported by the small $\chi$ value of 0.05 from the contact angle measurement. Importantly, based on the OOP (010) peak, the $d$-spacing value decreases $(3.70 \rightarrow 3.65 \AA)$ and the coherence length (CCL) value increases substantially from 22.16 to $24.58 \AA$ with the addition of $10 \%$ PM7-Si content, thus leading to the enhancement in crystallization and further assist charge transport and improve the device performance.

Atomic force microscopy (AFM) measurements were also carried out to probe the surface morphology of the different blend layers (Figure 5). Both the PM6:PM7-Si:C9 and PM6:C9 films exhibit fibrillar-like phase separation, while a larger domain-like phase separation is obtained in the binary PM7-Si:C9 film. Note that, the ternary PM6:PM7-Si:C9 film shows the smoothest surface with the root-mean-square (RMS) surface roughness value of $1.14 \mathrm{~nm}$, suggesting that the PM7-Si polymer mixes well in the ternary film. The AFM result further supports that the use of PM7-Si as a third component yields $\mathrm{BHJ}$ layers that are highly homogeneous and feature a fibrillar-network morphology, which is known to be associated with improved exciton dissociation, enhanced charge carrier transport, and ultimately higher cell efficiency. $^{41,68}$

\section{CONCLUSIONS}

In summary, a new donor polymer PM7-Si based on a BDT unit with chlorinated and alkylsilyl side chains was designed and synthesized as the third component for high-efficiency ternary PSCs. Owing to the structural similarity between PM6 and PM7-Si, the latter was found to be highly compatible with PM6 in ternary photoactive blends, as confirmed by contact angle measurements. Compared with binary devices, the ternary PSCs showed preferred energy alignment of frontier orbitals and improved charge transport properties; as a result, the ensuing PM6:PM7-Si:C9 cells exhibited a significantly improved PCE of $17.7 \%$. The improved efficiency was attributed to the simultaneous increase in $V_{\text {oc }}(0.864 \mathrm{~V}), J_{\mathrm{sc}}$ $\left(26.35 \mathrm{~mA} \mathrm{~cm}^{-2}\right)$, and FF $(77.6 \%)$, and is amongst the highest values reported to date for ternary PSCs. The improved $V_{\text {oc }}$ for optimized ternary PSC was found to be associated with a reduced $E_{\text {loss }}$ upon incorporation of PM7-Si. The present work demonstrates the potential of side-chain engineering to improve the $V_{\mathrm{oc}}$ and FF of PSCs without compromising the $J_{s c}$. It also provides valuable guidelines for the design of new donor materials specifically for boosting the performance of multicomponent PSCs.

\section{ASSOCIATED CONTENT}

\section{Supporting Information}

The Supporting Information is available free of charge at https://pubs.acs.org/doi/10.1021/acs.chemmater.1c01433.

Synthesis of polymer; ${ }^{1} \mathrm{H}$ NMR results; ${ }^{13} \mathrm{C}$ NMR results; device fabrication and characterization; GPC, $\mathrm{CV}$, photophysical, and electrochemical data; PL spectrum; contact angle and TOF-SIMS measurements; dark $J-V$ characteristics; and GIWAXS measurements (PDF) 


\section{AUTHOR INFORMATION}

\section{Corresponding Authors}

Yuanbao Lin - King Abdullah University of Science and Technology (KAUST), KAUST Solar Center (KSC), Thuwal 23955, Saudi Arabia; Email: yuanbao.lin@ kaust.edu.sa

Weiguo Zhu - School of Materials Science and Engineering, Jiangsu Engineering Laboratory of Light-Electricity-Heat Energy-Converting Materials and Applications, Jiangsu Collaborative Innovation Center of Photovoltaic Science and Engineering, Changzhou University, Changzhou 213164, China; (1) orcid.org/0000-0002-4244-2638; Email: zhuwg18@126.com

Thomas D. Anthopoulos - King Abdullah University of Science and Technology (KAUST), KAUST Solar Center (KSC), Thuwal 23955, Saudi Arabia; ○orcid.org/00000002-0978-8813; Email: thomas.anthopoulos@ kaust.edu.sa

Ergang Wang - Department of Chemistry and Chemical Engineering, Chalmers University of Technology, Göteborg SE-412 96, Sweden; School of Materials Science and Engineering, Zhengzhou University, Zhengzhou 450001, China; orcid.org/0000-0002-4942-3771;

Email: ergang@chalmers.se

\section{Authors}

Wenhong Peng - School of Materials Science and Engineering, Jiangsu Engineering Laboratory of Light-Electricity-Heat Energy-Converting Materials and Applications, Jiangsu Collaborative Innovation Center of Photovoltaic Science and Engineering, Changzhou University, Changzhou 213164, China; Department of Chemistry and Chemical Engineering, Chalmers University of Technology, Göteborg SE-412 96, Sweden; Hunan Provincial Key Laboratory of Environmental Catalysis \& Waste Re-cycling, School of Materials and Chemical Engineering, Hunan Institute of Engineering, Xiangtan 411104, China

Sang Young Jeong - Department of Chemistry, Korea University, Seoul 02841, Republic of Korea

Yuliar Firdaus - King Abdullah University of Science and Technology (KAUST), KAUST Solar Center (KSC), Thuwal 23955, Saudi Arabia; Research Center for Electronics and Telecommunication, Indonesian Institute of Science, Bandung 40135, Indonesia

Zewdneh Genene - Department of Chemistry and Chemical Engineering, Chalmers University of Technology, Göteborg SE-412 96, Sweden

Aggelos Nikitaras - Department of Physics, National Technical University of Athens, Athens GR-15780, Greece

Leonidas Tsetseris - Department of Physics, National Technical University of Athens, Athens GR-15780, Greece

Han Young Woo - Department of Chemistry, Korea University, Seoul 02841, Republic of Korea; (o orcid.org/ 0000-0001-5650-7482

Complete contact information is available at: https://pubs.acs.org/10.1021/acs.chemmater.1c01433

\section{Notes}

The authors declare no competing financial interest.

\section{ACKNOWLEDGMENTS}

The authors thank the Swedish Research Council (201606146, 2019-04683), the Swedish Research Council Formas, and the Knut and Alice Wallenberg Foundation (2017.0186, 2016.0059) for financial support. This work was also supported by the National Natural Science Foundation of China (51673031, 51573154), the Major Program of the Natural Science Research of Jiangsu Higher Education Institutions (18KJA480001), the Top-notch Academic Programs Project (TAPP) for Polymeric Materials Science and Engineering \& the Priority Academic Program Development (PAPD) of Jiangsu Higher Education Institutions, the Jiangsu Provincial Talents Project of High-Level Innovation and Entrepreneurship, and the Foundation of State Key Laboratory of Polymer Materials Engineering (sklpme2017-2-04). W.P. acknowledges the support by the China Scholarship Council and Y.L., A.N., L.T., and T.D.A. acknowledge the support by the King Abdullah University of Science and Technology (KAUST) Office of Sponsored Research (OSR) under Award No: OSR2018-CARF/CCF-3079 and No: OSR-2019-CRG8-4095.3. A.N. and L.T. acknowledge support for the computational time granted from GRNET in the National HPC facilityARIS- under project 9016-CREAM. H.Y.W acknowledges the financial support from the National Research Foundation (NRF) of Korea (2019R1A6A1A11044070).

\section{REFERENCES}

(1) Zheng, Z.; Yao, H.; Ye, L.; Xu, Y.; Zhang, S.; Hou, J. PBDB-T and its Derivatives: A Family of Polymer Donors Enables Over 17\% Efficiency in Organic Photovoltaics. Mater. Today 2020, 35, 115-130.

(2) Peng, W.; Zhang, G.; Shao, L.; Ma, C.; Zhang, B.; Chi, W.; Peng, Q.; Zhu, W. Simple-Structured Small Molecule Acceptors Constructed by A Weakly Electron-Deficient Thiazolothiazole Core for High-Efficiency Nonfullerene Organic Solar Cells. J. Mater. Chem. A 2018, 6, 24267-24276.

(3) Fan, Q.; Su, W.; Chen, S.; Kim, W.; Chen, X.; Lee, B.; Liu, T.; Romero, U. A. M.; Ma, R.; Yang, T.; Zhuang, W.; Li, Y.; Li, W.; Kim, T.-S.; Hou, L.; Yang, C.; Yan, H.; Yu, D.; Wang, E. Mechanically Robust All-Polymer Solar Cells from Narrow Band Gap Acceptors with Hetero-Bridging Atoms. Joule 2020, 4, 658-672.

(4) Sun, L.; Xu, X.; Song, S.; Zhang, Y.; Miao, C.; Liu, X.; Xing, G.; Zhang, S. Medium-Bandgap Conjugated Polymer Donors for Organic Photovoltaics. Macromol. Rapid Commun. 2019, No. 1900074.

(5) Fan, Q.; Romero, U. A. M.; Guo, X.; Wang, E.; Zhang, M.; Li, Y. Fluorinated Photovoltaic Materials for High-Performance Organic Solar Cells. Chem. - Asian J. 2019, 14, 3085-3095.

(6) Huang, F.; Bo, Z.-S.; Geng, Y.-H.; Wang, X.-H.; Wang, L.-X.; Ma, Y.-G.; Hou, J.-H.; Hu, W.-P.; Pei, J.; Dong, H.-L.; Wang, S.; Li, Z.; Shuai, Z.-G.; Li, Y.-F.; Cao, Y. Study on Optoelectronic Polymers: An Overview and Outlook. Acta Polym. Sin. 2019, 50, 988-1046.

(7) Jin, K.; Xiao, Z.; Ding, L. D18, An Eximious Solar Polymer! J. Semicond. 2021, 42, No. 010502.

(8) Li, S.; Li, C.-Z.; Shi, M.; Chen, H. New Phase for Organic Solar Cell Research: Emergence of Y-Series Electron Acceptors and Their Perspectives. ACS Energy Lett. 2020, 5, 1554-1567.

(9) Zhang, G.; Chen, X.-K.; Xiao, J.; Chow, P. C. Y.; Ren, M.; Kupgan, G.; Jiao, X.; Chan, C. C. S.; Du, X.; Xia, R.; Chen, Z.; Yuan, J.; Zhang, Y.; Zhang, S.; Liu, Y.; Zou, Y.; Yan, H.; Wong, K. S.; Coropceanu, V.; Li, N.; Brabec, C. J.; Bredas, J.-L.; Yip, H.-L.; Cao, Y. Delocalization of Exciton and Electron Wavefunction in Nonfullerene Acceptor Molecules Enables Efficient Organic Solar Cells. Nat. Commun. 2020, 11, No. 3943.

(10) Cui, Y.; Yao, H.; Zhang, J.; Xian, K.; Zhang, T.; Hong, L.; Wang, Y.; Xu, Y.; Ma, K.; An, C.; He, C.; Wei, Z.; Gao, F.; Hou, J. Single Junction Organic Photovoltaic Cells with Approaching 18\% Efficiency. Adv. Mater. 2020, 32, No. 1908205. 
(11) Lin, Y.; Firdaus, Y.; Isikgor, F. H.; Nugraha, M. I.; Yengel, E.; Harrison, G. T.; Hallani, R.; El-Labban, A.; Faber, H.; Ma, C.; Zheng, X.; Subbiah, A.; Howells, C. T.; Bakr, O. M.; McCulloch, I.; Wolf, S. D.; Tsetseris, L.; Anthopoulos, T. D. Self-Assembled Monolayer Enables Hole Transport Layer-Free Organic Solar Cells with 18\% Efficiency and Improved Operational Stability. ACS Energy Lett. 2020, 5, 2935-2944.

(12) Zhang, M.; Zhu, L.; Zhou, G.; Hao, T.; Qiu, C.; Zhao, Z.; Hu, Q.; Larson, B. W.; Zhu, H.; Ma, Z.; Tang, Z.; Feng, W.; Zhang, Y.; Russell, T. P.; Liu, F. Single-Layered Organic Photovoltaics with Double Cascading Charge Transport Pathways: 18\% Efficiencies. Nat. Commun. 2021, 12, No. 309.

(13) Qin, J.; Zhang, L.; Zuo, C.; Xiao, Z.; Yuan, Y.; Yang, S.; Hao, F.; Cheng, M.; Sun, K.; Bao, Q.; Bin, Z.; Jin, Z.; Ding, L. A Chlorinated Copolymer Donor Demonstrates a $18.13 \%$ Power Conversion Efficiency. J. Semicond. 2021, 42, No. 010501.

(14) Li, C.; Zhou, J.; Song, J.; Xu, J.; Zhang, H.; Zhang, X.; Guo, J.; Zhu, L.; Wei, D.; Han, G.; Min, J.; Zhang, Y.; Xie, Z.; Yi, Y.; Yan, H.; Gao, F.; Liu, F.; Sun, Y. Non-Fullerene Acceptors with Branched Side Chains and Improved Molecular Packing to Exceed 18\% Efficiency in Organic Solar Cells. Nat. Energy 2021, 6, 605-613.

(15) Liu, Q.; Jiang, Y.; Jin, K.; Qin, J.; Xu, J.; Li, W.; Xiong, W.; Liu, J.; Xiao, Z.; Sun, K.; Yang, S.; Zhang, X.; Ding, L. $18 \%$ Efficiency Organic Solar Cells. Sci. Bull. 2020, 65, 272-275.

(16) Sun, C.; Pan, F.; Qiu, B.; Qin, S.; Chen, S.; Shang, Z.; Meng, L.; Yang, C.; Li, Y. D-A Copolymer Donor Based on Bithienyl Benzodithiophene D-Unit and Monoalkoxy Bifluoroquinoxaline AUnit for High-Performance Polymer Solar Cells. Chem. Mater. 2020, 32, 3254-3261.

(17) Liu, F.; Xiao, C.; Feng, G.; Li, C.; Wu, Y.; Zhou, E.; Li, W. End Group Engineering on the Side Chains of Conjugated Polymers toward Efficient Non-Fullerene Organic Solar Cells. ACS Appl. Mater. Interfaces 2020, 12, 6151-6158.

(18) Fan, B.; Zhang, D.; Li, M.; Zhong, W.; Zeng, Z.; Ying, L.; Huang, F.; Cao, Y. Achieving Over 16\% Efficiency for Single-Junction Organic Solar Cells. Sci. China Chem. 2019, 62, 746-752.

(19) Hong, L.; Yao, H.; Wu, Z.; Cui, Y.; Zhang, T.; Xu, Y.; Yu, R.; Liao, Q.; Gao, B.; Xian, K.; Woo, H. Y.; Ge, Z.; Hou, J. EcoCompatible Solvent-Processed Organic Photovoltaic Cells with Over 16\% Efficiency. Adv. Mater. 2019, 31, No. 1903441.

(20) Chen, Y.; Geng, Y.; Tang, A.; Wang, X.; Sun, Y.; Zhou, E. Changing the $\pi$-Bridge from Thiophene to Thieno[3,2-b]thiophene for the D- $\pi-\mathrm{A}$ Type Polymer Enables High Performance FullereneFree Organic Solar Cells. Chem. Commun. 2019, 55, 6708-6710.

(21) Xu, X.; Feng, K.; Bi, Z.; Ma, W.; Zhang, G.; Peng, Q. SingleJunction Polymer Solar Cells with $16.35 \%$ Efficiency Enabled by a Platinum (II) Complexation Strategy. Adv. Mater. 2019, 31, No. 1901872.

(22) Wang, T.; Qin, J.; Xiao, Z.; Meng, X.; Zuo, C.; Yang, B.; Tan, H.; Yang, J.; Yang, S.; Sun, K.; Xie, S.; Ding, L. A $2.16 \mathrm{eV}$ Bandgap Polymer Donor Gives 16\% Power Conversion Efficiency. Sci. Bull. 2020, 65, 179-181.

(23) Lin, Y.; Firdaus, Y.; Nugraha, M. I.; Liu, F.; Karuthedath, S.; Emwas, A.-H.; Zhang, W.; Seitkhan, A.; Neophytou, M.; Faber, H.; Yengel, E.; McCulloch, I.; Tsetseris, L.; Laquai, F.; Anthopoulos, T. D. $17.1 \%$ Efficient Single-Junction Organic Solar Cells Enabled by nType Doping of the Bulk-Heterojunction. Adv. Sci. 2020, 7, No. 1903419.

(24) Lin, Y.; Adilbekova, B.; Firdaus, Y.; Yengel, E.; Faber, H.; Sajjad, M.; Zheng, X.; Yarali, E.; Seitkhan, A.; Bakr, O. M.; El-Labban, A.; Schwingenschlögl, U.; Tung, V.; McCulloch, I.; Laquai, F.; Anthopoulos, T. D. 17\% Efficient Organic Solar Cells Based on Liquid Exfoliated WS2 as a Replacement for PEDOT:PSS. Adv. Mater. 2019, 31, No. 1902965.

(25) Gasparini, N.; Salleo, A.; McCulloch, I.; Baran, D. The Role of the Third Component in Ternary Organic Solar Cells. Nat. Rev. Mater. 2019, 4, 229-242.

(26) Zhou, D.; You, W.; Xu, H.; Tong, Y.; Hu, B.; Xie, Y.; Chen, L. Recent Progress in Ternary Organic Solar Cells Based on Solution-
Processed Non-Fullerene Acceptors. J. Mater. Chem. A 2020, 8, 23096-23122.

(27) Khlyabich, P. P.; Burkhart, B.; Thompson, B. C. Efficient Ternary Blend Bulk Heterojunction Solar Cells with Tunable OpenCircuit Voltage. J. Am. Chem. Soc. 2011, 133, 14534-14537.

(28) Street, R. A.; Davies, R. A.; Khlyabich, P. P.; Burkhart, B.; Thompson, B. C. Origin of the Tunable Open-Circuit Voltage in Ternary Blend Bulk Heterojunction Organic Solar Cells. J. Am. Chem. Soc. 2013, 135, 986-989.

(29) Khlyabich, P. P.; Burkhart, P. P.; Thompson, B. C. Compositional Dependence of the Open-Circuit Voltage in Ternary Blend Bulk Heterojunction Solar Cells Based on Two Donor Polymers. J. Am. Chem. Soc. 2012, 134, 9074-9077.

(30) Khlyabich, P. P.; Rudenko, A. E.; Thompson, B. C.; Loo, Y.-L. Structural Origins for Tunable Open-Circuit Voltage in TernaryBlend Organic Solar Cells. Adv. Funct. Mater. 2015, 25, 5557-5563.

(31) Yu, R.; Yao, H.; Cui, Y.; Hong, L.; He, C.; Hou, J. Improved Charge Transport and Reduced Nonradiative Energy Loss Enable Over $16 \%$ Efficiency in Ternary Polymer Solar Cells. Adv. Mater. 2019, 31, No. 1902302.

(32) Zhan, L.; Li, S.; Xia, X.; Li, Y.; Lu, X.; Zuo, L.; Shi, M.; Chen, H. Layer-by-Layer Processed Ternary Organic Photovoltaics with Efficiency over 18\%. Adv. Mater. 2021, No. 2007231.

(33) Jin, K.; Xiao, Z.; Ding, L. 18.69\% PCE from Organic Solar Cells. J. Semicond. 2021, 42, No. 060502.

(34) Zhan, L.; Li, S.; Lau, T.-K.; Cui, Y.; Lu, X.; Shi, M.; Li, C.-Z.; Chen, H.; et al. Over 17\% Efficiency Ternary Organic Solar Cells Enabled by Two Non-Fullerene Acceptors Working in an Alloy-Like Model. Energy Environ. Sci. 2020, 13, 635-645.

(35) Tang, Y.; Yu, J.; Sun, H.; Wu, Z.; Koh, C. W.; Wu, X.; Liu, B.; Wang, J.; Liao, Q.; Li, Y.; Guo, H.; Woo, H. Y.; Gao, F.; Guo, X. Two Compatible Polymer Donors Enabling Ternary Organic Solar Cells with a Small Nonradiative Energy Loss and Broad Composition Tolerance. Sol. RRL 2020, 4, No. 2000396.

(36) Zhang, Y.; Liu, D.; Lau, T. K.; Zhan, L.; Shen, D.; Fong, P. W. K.; Yan, C.; Zhang, S.; Lu, X.; Lee, C. S.; Hou, J.; Chen, H.; Li, G. A Novel Wide-Bandgap Polymer with Deep Ionization Potential Enables Exceeding 16\% Efficiency in Ternary Nonfullerene Polymer Solar Cells. Adv. Funct. Mater. 2020, 30, No. 1910466.

(37) Fan, Q.; Su, W.; Wang, Y.; Guo, B.; Jiang, Y.; Guo, X.; Liu, F.; Russell, T. P.; Zhang, M.; Li, Y. Synergistic Effect of Fluorination on Both Donor and Acceptor Materials for High Performance NonFullerene Polymer Solar Cells with $13.5 \%$ Efficiency. Sci. China: Chem. 2018, 5, 531-537.

(38) Song, J.; Li, C.; Zhu, L.; Guo, J.; Xu, J.; Zhang, X.; Weng, K.; Zhang, K.; Min, J.; Hao, X.; Zhang, Y.; Liu, F.; Sun, Y. Ternary Organic Solar Cells with Efficiency>16.5\% Based on Two Compatible Nonfullerene Acceptors. Adv. Mater. 2019, 31, No. 1905645.

(39) Su, W.; Fan, Q.; Guo, X.; Meng, X.; Bi, Z.; Ma, W.; Zhang, M.; Li, Y. Two Compatible Nonfullerene Acceptors with Similar Structures as Alloy for Efficient Ternary Polymer Solar Cells. Nano Energy 2017, 38, 510-517.

(40) Hu, Z.; Yang, L.; Gao, W.; Gao, J.; Xu, C.; Zhang, X.; Wang, Z.; Tang, Z.; Yang, C.; Zhang, F. Over $15.7 \%$ Efficiency of Ternary Organic Solar Cells by Employing Two Compatible Acceptors with Similar LUMO Levels. Small 2020, 16, No. 2000441.

(41) An, Q.; Wang, J.; Ma, X.; Gao, J.; Hu, Z.; Liu, B.; Sun, H.; Guo, X.; Zhang, X.; Zhang, F. Two Compatible Polymer Donors Contribute Synergistically for Ternary Organic Solar Cells with 17.53\% Efficiency. Energy Environ. Sci. 2020, 13, 5039-5047.

(42) Su, W.; Li, G.; Fan, Q.; Zhu, Q.; Guo, X.; Chen, J.; Wu, J.; Ma, W.; Zhang, M.; Li, Y. Nonhalogen Solvent-Processed Polymer Solar Cells Based on Chlorine and Trialkylsilyl Substituted Conjugated Polymers Achieve 12.8\% Efficiency. J. Mater. Chem. A 2019, 7, 23512359.

(43) Bin, H.; Gao, L.; Zhang, Z.-G.; Yang, Y.; Zhang, Y.; Zhang, C.; Chen, S.; Xue, L.; Yang, C.; Xiao, M.; Li, Y. 11.4\% Efficiency NonFullerene Polymer Solar Cells with Trialkylsilyl Substituted 2DConjugated Polymer as Donor. Nat. Commun. 2016, 7, No. 13651. 
(44) Yao, H.; Wang, J.; Xu, Y.; Zhang, S.; Hou, J. Recent Progress in Chlorinated Organic Photovoltaic Materials. Acc. Chem. Res. 2020, 53, $822-832$.

(45) Valiev, M.; Bylaska, E. J.; Govind, N.; Kowalski, K.; Straatsma, T. P.; Van Dam, H. J. J.; Wang, D.; Nieplocha, J.; Apra, E.; Windus, T. L.; de Jong, W. NWChem: A Comprehensive and Scalable OpenSource Solution for Large Scale Molecular Simulations. Comput. Phys. Commun. 2010, 181, 1477-1489.

(46) Becke, A. D. Density-Functional Thermochemistry. III. The Role of Exact Exchange. J. Chem. Phys. 1993, 98, 5648-5652.

(47) Stephens, P. J.; Devlin, F. J.; Chabalowski, C. F.; Frisch, M. J. Ab-Initio Calculation of Vibrational Absorption and CircularDichroism Spectra Using Density-Functional Force-Fields. J. Phys. Chem. A 1994, 98, 11623-11627.

(48) Godbout, N.; Salahub, D. R.; Andzelm, J.; Wimmer, E. Optimization of Gaussian-Type Basis Sets for Local Spin Density Functional Calculations. Part I. Boron Through Neon, Optimization Technique and Validation. Can. J. Chem. 1992, 70, 560-571.

(49) Momma, K.; Izumi, F. VESTA 3 for Three-Dimensional Visualization of Crystal, Volumetric and Morphology Data. J. Appl. Crystallogr. 2011, 44, 1272-1276.

(50) Hirata, S.; Head-Gordon, M. Time-Dependent Density Functional Theory within the Tamm-Dancoff Approximation. Chem. Phys. Lett. 1999, 314, 291-299.

(51) Zhang, W.; Huang, J.; Xu, J.; Han, M.; Su, D.; Wu, N.; Zhang, C.; Xu, A.; Zhan, C. Phthalimide Polymer Donor Guests Enable over 17\% Efficient Organic Solar Cells via Parallel-Like Ternary and Quaternary Strategies. Adv. Energy Mater. 2020, 10, No. 2001436.

(52) Gasparini, N.; Paleti, S. H. K.; Bertrandie, J.; Cai, G.; Zhang, G.; Wadsworth, A.; Lu, X.; Yip, H.-L.; McCulloch, I.; Baran, D. Exploiting Ternary Blends for Improved Photostability in High-Efficiency Organic Solar Cells. ACS Energy Lett. 2020, 5, 1371.

(53) Li, D.; Zhu, L.; Liu, X.; Xiao, W.; Yang, J.; Ma, R.; Ding, L.; Liu, F.; Duan, C.; Fahlman, M.; Bao, Q. Enhanced and Balanced Charge Transport Boosting Ternary Solar Cells Over 17\% Efficiency. Adv. Mater. 2020, 32, No. 2002344.

(54) An, Q.; Wang, J.; Gao, W.; Ma, X.; Hu, Z.; Gao, J.; Xu, C.; Hao, M.; Zhang, X.; Yang, C.; Zhang, F. Alloy-Like Ternary Polymer Solar Cells with Over 17.2\% Efficiency. Sci. Bull. 2020, 65, 538.

(55) Wang, R.; Zhang, C.; Li, Q.; Zhang, Z.; Wang, X.; Xiao, M. Charge Separation from an Intra-Moiety Intermediate State in the High-Performance PM6:Y6 Organic Photovoltaic Blend. J. Am. Chem. Soc. 2020, 142, 12751-12759.

(56) Kaelble, D. H. Dispersion-Polar Surface Tension Properties of Organic Solids. J. Adhes. 1970, 2, 66-81.

(57) Wang, H.; Yang, L.; Lin, P.-C.; Chueh, C.-C.; Liu, X.; Qu, S.; Guang, S.; Yu, J.; Tang, W. A Simple Dithieno[3,2-b:2',3'-d]pyrrolRhodanine Molecular Third Component Enables Over 16.7\% Efficiency and Stable Organic Solar Cells. Small 2021, 17, No. 2007746

(58) Lin, Y.; Jin, Y.; Dong, S.; Zheng, W.; Yang, J.; Liu, A.; Liu, F.; Jiang, Y.; P Russell, T.; Zhang, F.; Huang, F.; Hou, L. Printed Nonfullerene Organic Solar Cells with the Highest Efficiency of 9.5\%. Adv. Energy Mater. 2018, No. 1701942.

(59) Jiang, C. Y.; Chellappana, V.; Goha, W. P.; Zhang, J. Investigating Coating Method Induced Vertical Phase Distribution in Polymer-Fullerene Organic Solar Cells. Sol. Energy Mater. Sol. Cells 2018, 179, 241-246.

(60) Zhang, M.; Ming, R.; Gao, W.; An, Q.; Ma, X.; Hu, Z.; Yang, C.; Zhang, F. Ternary Polymer Solar Cells with Alloyed NonFullerene Acceptor Exhibiting 12.99\% Efficiency and 76.03\% Fill Factor. Nano Energy 2019, 59, 58-65.

(61) Wan, J.; Zhang, L.; He, Q.; Liu, S.; Huang, B.; Hu, L.; Zhou, W.; Chen, Y. High-Performance Pseudoplanar Heterojunction Ternary Organic Solar Cells with Nonfullerene Alloyed Acceptor. Adv. Funct. Mater. 2020, 30, No. 1909760.

(62) An, Q.; Wang, J.; Zhang, F. Ternary Polymer Solar Cells with Alloyed Donor Achieving 14.13\% Efficiency and 78.4\% Fill Factor. Nano Energy 2019, 60, 768-774.
(63) Lin, Y.; Dong, S.; Li, Z.; Zheng, W.; Yang, J.; Liu, A.; Cai, W.; Liu, F.; Jiang, Y.; P Russell, T.; Huang, F.; Wang, E.; Hou, L. EnergyEffectively Printed All-Polymer Solar Cells Exceeding 8.61\% Efficiency. Nano Energy 2018, 46, 428-435.

(64) Zhou, K.; Wu, Y.; Liu, Y.; Zhou, X.; Zhang, L.; Ma, W. Molecular Orientation of Polymer Acceptor Dominates Open-Circuit Voltage Losses in All-Polymer Solar Cells. ACS Energy Lett. 2019, 4, $1057-1064$.

(65) Zhang, Z.; Wang, H.; Yu, J.; Sun, R.; Xu, J.; Yang, L.; Geng, R.; Cao, J.; Du, F.; Min, J.; Liu, F.; Tang, W. Modification on the Indacenodithieno[3,2-b]thiophene Core to Achieve Higher Current and Reduced Energy Loss for Nonfullerene Solar Cells. Chem. Mater. 2020, 32, 1297-1307.

(66) Yue, Q.; Wu, H.; Zhou, Z.; Zhang, M.; Liu, F.; Zhu, X. 13.7\% Efficiency Small-Molecule Solar Cells Enabled by a Combination of Material and Morphology Optimization. Adv. Mater. 2019, 31, No. 1904283.

(67) Dai, T.; Li, X.; Zhang, Y.; Xu, D.; Geng, A.; Zhao, J.; Chen, X. Performance Improvement of Polymer Solar Cells with Binary Additives Induced Morphology Optimization and Interface Modification Simultaneously. Sol. Energy 2020, 201, 330-338.

(68) Xu, X.; Feng, K.; Lee, Y. W.; Woo, H. Y.; Zhang, G.; Peng, Q. Subtle Polymer Donor and Molecular Acceptor Design Enable Efficient Polymer Solar Cells with a Very Small Energy Loss. Adv. Funct. Mater. 2020, 30, No. 1907570. 